\title{
Platelets augment respiratory burst in neutrophils activated by selected species of gram-positive or gram-negative bacteria
}

\author{
Jacek Międzobrodzki', Tomasz Panz², Przemysław M. Płonka², Katarzyna Zając', \\ Joanna Dracz ${ }^{1}$, Kamila Pytel ${ }^{1}$, Lukasz Mateuszuk ${ }^{1,3}$, Stefan Chłopicki ${ }^{3}$ \\ ${ }^{1}$ Department of Microbiology, Faculty of Biochemistry, Biophysics and Biotechnology, \\ Jagiellonian University, Kraków, Poland \\ ${ }^{2}$ Department of Biophysics, Faculty of Biochemistry, Biophysics and Biotechnology, \\ Jagiellonian University, Kraków, Poland \\ ${ }^{3}$ Department of Experimental Pharmacology, Collegium Medicum, Jagiellonian University, Kraków, Poland
}

\begin{abstract}
Neutrophils and platelets circulate in blood system and play important physiological roles as part of immunological system. Neutrophils are the first line of host defense against various intruders, and platelets are satellite cells cooperating with other components of defense system. Recent studies report about the cooperation among these types of cells. We analyzed the effect of platelets on oxygen burst in neutrophils triggered by Staphylococcus aureus and Escherichia coli bacteria in vitro. The effect of platelets on oxygen burst in neutrophils was measured by luminol enhanced chemiluminescence. Opsonized and non-opsonized bacteria were used as activators. Activation of neutrophils with live non-opsonized and opsonized bacteria in the presence of platelets increased the oxygen burst as compared to the same system without platelets. The gram-positive bacteria (Staphylococcus aureus) were causing higher activation than gram-negative bacteria (Escherichia coli). This work demonstrate that platelets potentate the response of neutrophils augmenting their respiratory burst in vitro when triggered by bacteria.
\end{abstract}

Key words: Inflammation - Neutrophil - Platelet - Chemiluminescence - Oxygen burst - Bacteria - Staphylococcus aureus - Escherichia coli

\section{Introduction}

The inflammation is a complex cascade of cellular events leading to activation of some types of blood cells by other cells within positive feedback loop. Activated neutrophils release reactive oxygen species (ROS) or platelet-activating factor (PAF) [1,2]. Recently, an important role of platelets and their contribution to inflammation cascade have been recognized, as they contributed in secretion of a the following compounds: ADP, ATP, PDGF, PF4, PSGL-1, TXA2. Moreover, platelets interact with neutrophils [3]. Leukotriens, cytokines and hydrolases belong to the wide spectrum of physiological platelet activators. On the other hand,

Correspondence: J. Międzobrodzki, Jagiellonian University

Campus III, Department of Biochemistry, Biophysics and Biotechnology, Gronostajowa 7, 30-387 Kraków, Poland; tel.: (+4812) 6646371, fax.: (+4812) 6646902,

e-mail: jacekmie@mol.uj.edu.pl platelets produce ROS on various pathways: using membrane oxidases, NO synthesis, initiating metabolism of arachidonic acid, phosphoinositol, and glutathione cycle. The process of platelet activation is initiated, among the other, by: trombins, collagen, tromboxan A2, ADP. Most of these factors interact with receptors located on the platelet surface [4-6].

\footnotetext{
Abbreviations: ADP: adenosine diphosphate; ATP: adenosine triphosphate; BPI: bactericidal permeability increasing; CD: cluster of differentiation; CL: chemiluminescence; Gp: glycoprotein; HLA: human leukocyte antigen; LBP: lipopolysaccharide binding protein; LPS: lipopolysaccharide; $\mathrm{LTC}_{4}$ : leukotriene $\mathrm{C}$ four; $\mathrm{Lx}$ : latex, polystyrene particles; NADPH: nicotinamide adenine dinucleotide phosphate; NO: nitric oxygen; PAF: platelet activating factor; PBS: phosphate buffered saline; PDGF: platelet derived growth factor; $\mathrm{PF}_{4}$ : platelet factor four; PSGL-1: P-selectin glycoprotein ligand one; RLU: relative luminescence unit; ROS: reactive oxygen species; TLR: toll-like receptor; TSB: tryptic soy broth; $\mathrm{TXA}_{2}$ : tromboxan A two; v/v: volume/volume
} 
Platelets and neutrophils are able to create aggregates, due to expression of platelet-derived selectin-P and its ligand PSGL-1, and by fibrinogen bridges between platelet glycoprotein Gp IIb/IIIa and neutrophilic CD11b/CD18. These types of junctions may facilitate interactions between the both types of cells [7]. The interaction between phagocytic cells and bacteria induces activation of membrane oxidase which in turn triggers a metabolic response as respiratory burst in phagocyte. A number of ROS such as singlet oxygen, hydroxyl radical, superoxide anion are formed during that process [8]. In the presence of luminol, ROS cause the emission of light [9]. It is possible that platelets communicate with other cells of immune system changing activity of their NADPH oxidase [10]. In this work bacteria as factor affecting the activation of neutrophils either alone or cooperating with platelets were studied.

\section{Materials and methods}

Preparation of neutrophils and platelets. The study was carried with human neutrophils and platelets obtained from healthy donors. The bacteria strains used for the study were representing gram-negative and gram-positive bacteria, they were chosen from typical oportunistic bacteria of man. Blood donors were selected from the group of volunteers registered in blood bank of the university hospital, Jagiellonian Univerisity, Kraków. They did not take any medicines for at least two weeks before donation. The saturated solution of sodium citrate $(3.2 \%)$ was added to venous blood samples as anticoagulator $(1: 9 \mathrm{v} / \mathrm{v})$. Neutrophils and platelets were isolated from blood samples as described in our previous work [10]. Single chemiluminescence measurement was perfomed using neutrophils and platelets from the same donor, to avoid cross stimulation by HLA antigens.

Neutrophils. The blood was sedimented in the presence of dextran for $30 \mathrm{~min}$. ( $3 \%$ solution of dextran, 1:1 v/v) followed by FicollHypaque gradient to separate neutrophils from other leukocytes. Then after hypotonic lysis neutrophils were washed and resuspended in PBS with $0.1 \%$ glucose, and stored in $4{ }^{\circ} \mathrm{C}$ prior to experiments. More than $95 \%$ of the isolated cells were neutrophils due to microscope evaluation. Cells were viable in more than $98 \%$ as evidenced by trypan blue staining.

Platelets. Platelets were separated and purified according to Radomski and Moncada method [11]. In brief, blood was centrifuged at $250 \mathrm{~g}$ for $20 \mathrm{~min}$ to obtain platelet-rich plasma. Plateletpoor plasma was obtained by centrifugation of remaining blood for $5 \mathrm{~min}$ at $2.000 \mathrm{~g}$. Then platelets were washed twice in prostaglandin I2, and suspended in Ca-free PBS. Filtered platelets were obtained by gel filtration on Sepharose 2B columns.

Bacteria. Both Staphylococcus aureus and Escherichia coli strains were isolated from patients in Center of Microbiological Research and Autovaccines in Kraków, and identified in routine procedure using standard biochemical API bio-Merieux tests. Before the experiments bacteria were cultured overnight in TSB, Difco medium. The inoculum volume was kept low, to obtain bacteria for measurements in logarithmic growth phase. Cell suspensions were centrifuged and washed in PBS three times, stained and counted in Bürker chamber.

Opsonization. Opsonization of bacteria with serum proteins facilitates phagocytosis by neutrophils. Serum originated from the same healthy donnor, and the opsonization process was performed according to standard method. Briefly: $1 \mathrm{ml}$ of bacteria suspension in saline was supplemented with $100 \mu \mathrm{l}$ of serum, incubated $30 \mathrm{~min}$. at $37^{\circ} \mathrm{C}$, and then centrifuged $10 \mathrm{~min}$. at $70 \mathrm{~g}$. Supernatants were discarded, and cells in pellets were resuspended in $0.9 \%$ saline. Washing by centrifuging and suspending were repeated three times.

Reagents. All chemicals used in assays were of the highest commercially available purity. Latex, polystyrene beads, of diameter $0.9 \mu \mathrm{m}$ were from Institute of Catalysis and Physicochemistry, Polish Academy of Sciences in Kraków, Poland. A 10\% of standard latex suspension in volume $10 \mu \mathrm{l}$ was used in activation of cells.

Cell chemiluminescence $(C L)$ measurements. Light is produced as a result of release of excited species generated as products of oxygenation. Chemiluminometry can be succesfully used with the addition of luminol as chemiluminogenic probe for enhancing light emission [9]. Microplate CL assays have been used to analyze oxygen burst in neutrophil-platelet-bacteria experimental tests [12]. The CL signals were evaluated using a Berthold EG\&G model LB 96 P (Austria) automatic luminescence analyzer. WINGLOW programme provided by the luminescence analyzer manufacturer was used to evaluate the CL signals from each sample which was measured for $40 \mathrm{~min}$. at $37^{\circ} \mathrm{C}$. Isolated neutrophils, platelets, $\mathrm{CaCl}_{2}$, and suspensions of particular bacteria were recombined in selected positions of 96-wells milky-white microtiter plates (Nunc) in following order: $150 \mu \mathrm{l}$ of luminol (5-amino-2,3-dihydro-1,4-phtalazinedione) (Serva) in Krebs buffer; $50 \mu$ of neutrophils suspended in PBS at concentration of $2 \times 10^{3} / \mathrm{ml}, 25 \mu 1$ of platelets suspended in PBS at concentration of $2 \times 10^{6} / 1 \mathrm{ml}, 2 \mu \mathrm{l}$ of $\mathrm{CaCl}_{2} 90$ $\mathrm{vM}$, and tested bacteria suspension, as it was described previously $[10,13]$. Data were expressed as counts per minute (cpm) and plotted. The levels of CL were presented in relative luminescence units (RLU) after integration of the signals. Preliminary experiments were performed using different ratios of bacteria to neutrophils and platelets to neutrophils (data not shown), but finally neutrophil to platelet ratio was kept 1:500, and platelets to bacteria ratio was kept 1:10. All measurements were run in duplicates.

Statistical analysis. The luminol enhanced chemiluminescence integral caused by neutrophils in presence of platelets and various bacteria samples were analyzed. The data shown presents the mean of at least three measurements with standard deviation error bars.

\section{Results}

\section{The chemiluminescence (CL) of neutrophils and platelets stimulated with gram-positive bacteria $S$. aureus}

The addition of the live non-opsonized bacteria $S$. aureus to the samples containing neutrophils caused increase of the chemiluminescence by about $20 \%$ when compared with non-activated neutrophils (Fig. 1a). The addition of platelets augmented the response by about $100 \%$ in comparison with neutrophils in the presence of bacteria alone. The addition of live and opsonized bacteria $S$. aureus to the sample of neutrophils reaching the level three-fold higher then of CL of non-activated neutrophils (Fig. 1b). The addition of platelets potentates the effect to reach approximately four-fold increase of neutrophil response in comparison with non-stimulated neutrophils. 

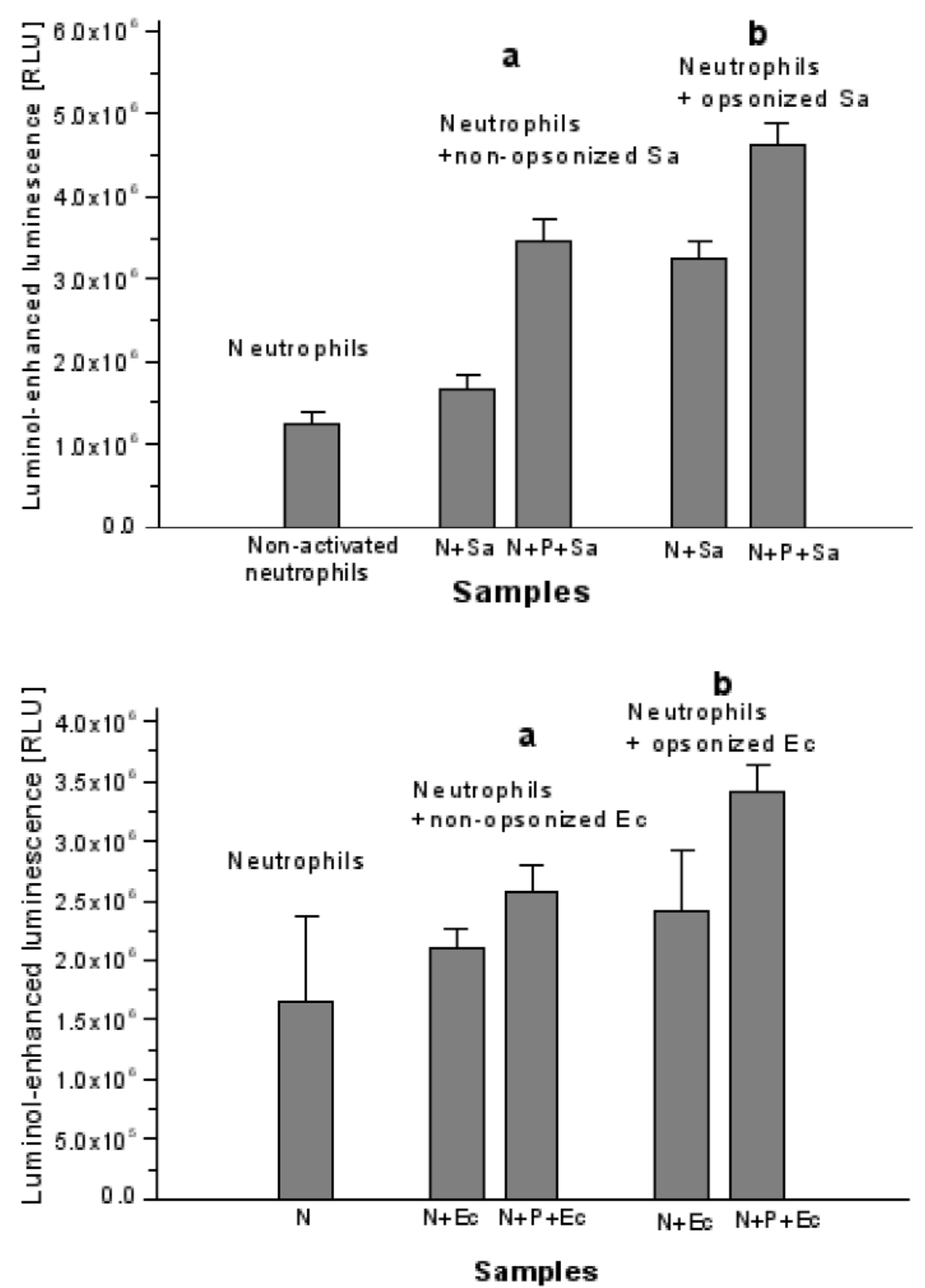

Fig. 1. Chemiluminescence of platelets and neutrophils stimulated by live Staphylococcus aureus bacteria (N - neutrophils, P - platelets, $\mathrm{Sa}$ - Staphylococcus aureus cells).
Fig. 2. Chemiluminescence of platelets and neutrophils stimulated by live Escherichia coli bacteria (N neutrophils, P - platelets, Ec Escherichia coli cells).

\section{The chemiluminescence (CL) of neutrophils and platelets stimulated with gram-negative bacteria $E$. coli}

The non-opsonized bacteria $E$. coli alone did not increase the level of luminol-enhanced CL (Fig. 2a, left profile) in statistically significant manner. The neutrophils with platelets treated with non-opsonized E. coli bacteria have shown the slight but statistically significant increase of CL (Fig. 2a, right profile).

The live opsonized gram-negative $E$. coli caused the increase of oxygen burst of neutrophils as shown on Fig. 2b. In the presence of platelets neutrophils responded to the presence of bacteria with slightly higher CL level. It should be noticed that the CL level of neutrophils treated with non-opsonized bacteria was lower than the level of CL recorded for neutrophils treated with opsonized bacteria.

The control experiment in which the CL level of platelets alone in the presence of different activators (latex and bacteria samples) indicated that CL was an order of magnitude weaker than the CL signals from neutrophils (Fig. 3 and Fig. 4, left profiles). The increase of the CL signal from platelets after treatment with latex was about three-times higher than from platelets without activator. Addition of bacteria to the platelets did not caused any significant increase of CL. This phenomenon was recorded for both 


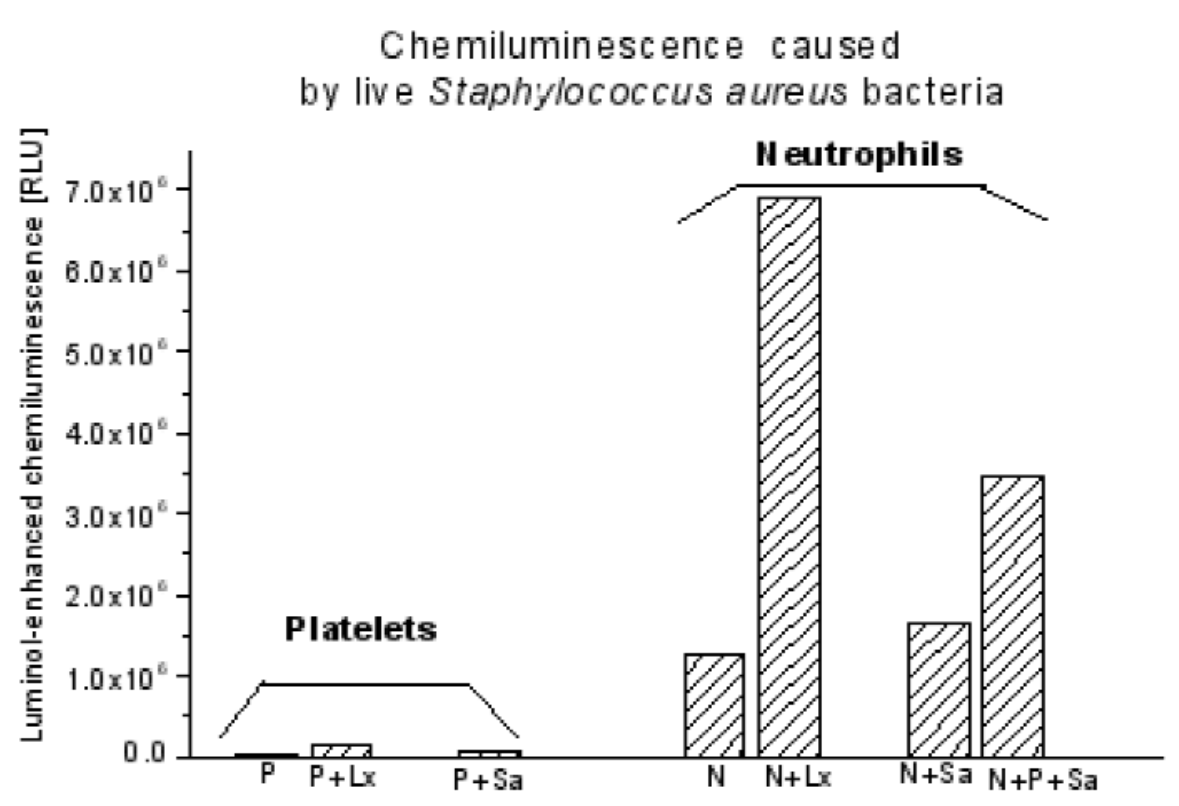

Chemiluminescence caused

by live Escherichia coli bacteria

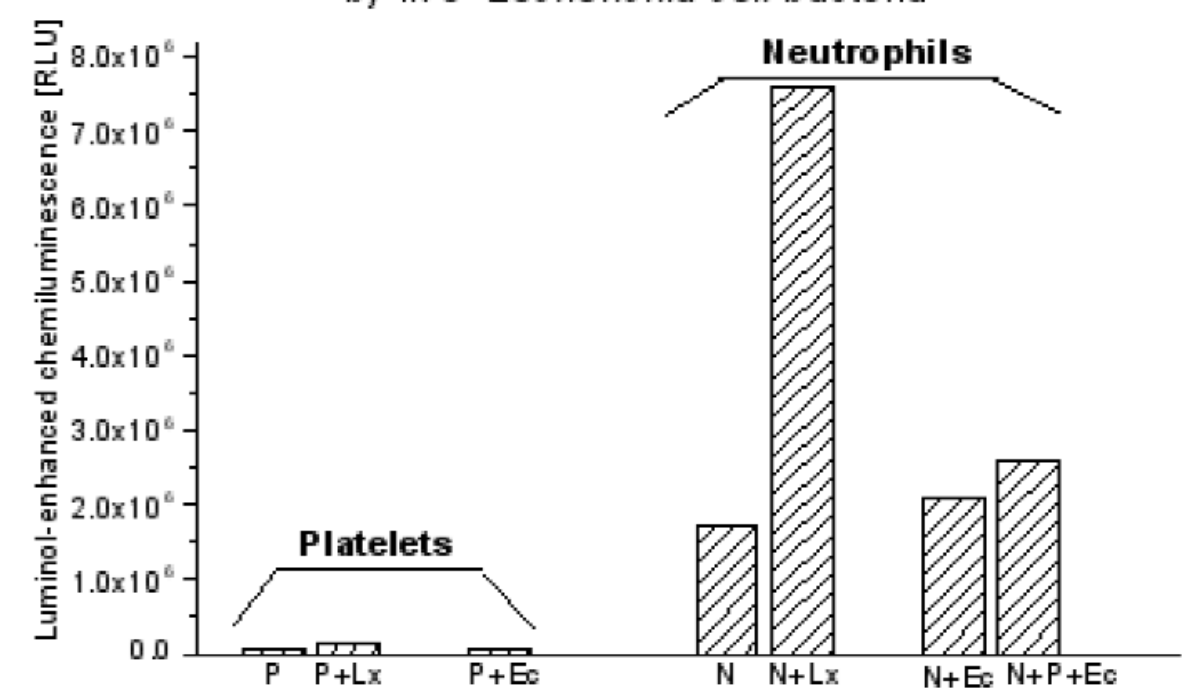

Fig. 3. Examples of influence of platelets on neutrophils response triggered by opsonized Staphylococcus aureus bacteria (P - platelets, Lx latex, polystyrene beads, Sa - Staphylococcus aureus cells, $\mathrm{N}$ - neutrophils).

Fig. 4. Examples of influence of platelets on neutrophils response triggered by non-opsonized Escherichia coli bacteria ( $\mathrm{P}$ - platelets, $\mathrm{Lx}$ - latex, polystyrene beads, N - neutrophils, Ec - Escherichia coli cells).

gram-positive (Fig. 3), and gram-negative (Fig. 4) bacteria.

The influence of platelets on oxygen burst, measured with luminol enhanced luminescence of bacteria activated neutrophils, was analyzed. The results indicate statistically significant increase activation in the presence of platelets.

\section{Discussion}

A complex interactions between neutrophils and platelets take place during imflammation. Factors originating from both types of cells form complicated sys- tem of physiological stimulation, known as "cell crosstalk" [2,3]. The detailed molecular mechanisms of these interactions are not known yet. The already published data show that neutrophils can affect the adhesion, aggregation and secretion of platelets [14]. The other results show the influence of platelets on chemotaxis, phagocytosis, degranulation and oxygen metabolism of neutrophils $[7,15,16]$. The key factors engaged in these interactions are lipid mediators as: PAF, LTC4, TXA2, and others $[3,6]$. These mediators appear to augment cell-cell interaction and together with selectin-P take part in regulation of platelets adhesion to neutrophils $[10,17]$. Basing on these find- 
ings, the problem of oxygen burst caused by bacteria in the presence of cell cross-talk partners, namely neutrophils and platelets, may provide new data for explanation of physiology of inflammation.

It was already shown that the commonly used activator latex (polystyrene beads) or bacteria stimulate CL response from neutrophils, and presence of platelets additionally augment oxygen burst [10]. The results obtained for Staphylococcus aureus bacteria as activating factor for CL is shown in Fig .1, and the effect of Escherichia coli bacteria, possessing other than $S$. aureus chemical structure of cell wall, is shown in Fig. 2. Use of $S$. aureus bacteria as activators was more effective in stimulating the reactive oxygen species for opsonized cells than for non-opsonized ones. This effect might be seen for both neutrophils and for platelets.

The interaction between phagocytic cells and bacteria induces activation of membrane oxidase which triggers respiratory burst in phagocyte. The bacteria are both physiological and pathological activators of oxygen burst in host organism cells. The various bacteria differ with the structure of cell wall, which affect the level of oxygen burst in neutrophils and platelets separately, as well as in cooperating cells. Gram-positive bacteria, $S$. aureus, live and opsonized, caused three-fold stronger oxygen burst, whereas gram-negative E. coli, live and opsonized, did not cause slighter increase of oxygen burst in neutrophils and platelets.

When non-opsonized bacteria were used, only gram-positive $S$. aureus triggered 2.5 times stronger oxygen burst when compared with non-activated neutrophils. The specific compound of cell wall of grampositive bacteria, namely peptidoglycane, is recognized by neutrophils with specific receptors. One group of these receptors are the proteins recognizing peptidoglycane. The high level of mRNA for these proteins was detected in neutrophils. The proteins recognizing peptidoglycan are functional as intracellular antibacterial proteins in neutrophils and inhibit the growth of gram-positive bacteria, and contribute to the inhibition of oxygen burst in neutrophils [17].

Briefly, the performed experiments have shown that gram-positive $S$. aureus bacteria activate neutrophils and that platelets augment that process leading to producing the reactive oxygen species (ROS). The result of platelet and neutrophil interaction is strong oxygen burst. It was confirmed that opsonized $S$. aureus bacteria caused the higher production of reactive oxygen species in studied cells (neutrophils and platelets) than non-opsonized bacteria.

It is postulated that for activation of neutrophils by gram-negative bacteria e.g. E. coli the key role is played by lipopolysaccharide (LPS), which is the bacterial endotoxin present in the cell wall [18]. After lysis of bacteria LPS is released from the cell wall. It enters the circulation and it is bound by serum protein LBP, which in turn transports LPS to the target cells (neutrophils, monocytes) presenting it to either CD14 or TLR-2 [19].

Consistently, it might be noticed, that platelets are less effective in stimulation of gram-negative bacteria measured with CL than the effect of platelet augumented stimulation of gram-positive bacteria (Figs. 3 and 4 , right profiles).

As the result of this sequence of events, the expression of the molecules co-stimulating the oxygen consumption by neutrophils takes place [18]. The stimulation of neutrophils with opsonized bacteria caused the stronger response than stimulation with nonopsonized bacteria. The serum used for opsonization contained the proteins released by complement system triggered by LPS. This activation triggered the cascade of proteolytic reactions leading to production of factors exciting the cells of immune system for phagocytosis [21]. In this cascade bacteria opsonized by serum IgG antibodies and $\mathrm{C} 3 \mathrm{~b}$ complement fragment are recognized by phagocytic cells and phagocytosed. This triggers activation of NADPH-oxidase and production of ROS.

In summary, the opsonized E. coli gram-negative bacteria are moderate stimulators of neutrophils and platelets. The non-opsonized bacteria stimulate these cells only slightly.

The last decade has brought several findings about the function of platelets, as stimulators of phagocytic activity, synthesis of NADPH oxidase and generation of reactive oxygen species (ROS) [10,22]. The reactive oxygen species, easily detected with CL method, might play a key role in the cooperation of neutrophils with platelets, which is followed by increased mass production of ROS in neutrophils [23]. The main lipidic mediator released by platelets is tromboxane, therefore it is possible that TXA2 affects the oxygen metabolism of neutrophils, which lack this enzyme [24]. The proposed order of events assumes significant role of platelets in activation of neutrophils, and it might be useful as starting point for studies of molecular mechanisms of inflammation process caused by bacterial infection.

Acknowledgments: The authors are indebted to Dr. Andrzej Kasprowicz and Dr. Anna Bialecka from Centre of Microbiological Research and Autovaccines in Kraków for providing the bacteria strains, and to Jolanta Reyman and Agnieszka Bukowiec for their excellent technical assistance. That project was supported by the grant No. PBZ-KBN-101/T09/2003/14 awarded to J.M. from Polish State Committee for Scientific Research KBN.

\section{References}

[1] Colli S, Eligini S, Lalli M, Tremoli E. Platelet-neutrophil interactions and superoxide anion generation: involvement of purine nucleocides. Free Rad Biol Med. 1996;20:271-278. 
[2] Selak MA. Neutrophil-platelet interactions in inflammation. Receptor. 1994;4:3-7.

[3] Heffner JE. Platelet-neutrophil interactions in sepsis - platelet guilt by association? Intensive Care Med. 1997;23:366-368.

[4] Lopez-Farre A, Caramelo C, Esteban A, Alberola ML, Millas I, Monton M, Casado S. Effects of Aspirin on Platelet-Neutrophil Interactions. Role of Nitric Oxide and Endothelin-1. Circulation. 1995;91:2080-20884.

[5] Olas B, Wachowicz B. Role of reactive oxygen species in blood platelets. Post Biol Kom. 2003;30:325-333.

[6] Weyrich AS, Zimmerman GA. Platelets: signaling cells in the immune continuum. Trends Immunol. 2004;25:489-495.

[ 7] Li N, Hu H, Lindquist M, Wikstrom-Jonsson E, Goodall AH, Hjemdahl P. Platelet-Leukocyte Cross Talk in Whole Blood. Arterioscler Thromb Vasc Biol. 2000;20:2702-2708.

[ 8] Carulli G, Barsotti G, Cupisti A, Minnucci S, Gianfaldoni ML, Agostini B, Ambrogi F. Platelet-Neutrophil Interactions in Uremic Patients: Effects on Neutrophil Superoxide Anion production and Chemiluminescence. Nephron. 1995;69:248-252.

[9] Robinson P, Wakefield D, Breit SN, Easter JF, Penny R. Chemiluminescent response to pathogenic organisms: normal human polymorphonuclear leukocytes. Infect Immunol. 1984; 43:744-752.

[10] Chlopicki S, Olszanecki R, Janiszewski M, Laurindo FRM, Panz T, Miedzobrodzki J. Functional Role of NADPH Oxidase in Activation of Platelets. Antiox Redox Signal. 2004;6: 691-698.

[11] Radomski M, Moncada S. An improved method for washing of human platelets with prostacyclin. Thromb Res. 1983;30: 383-389.

[12] Kournikakis B, Simpson M. Optimization of a Phagocyte Microplate Chemiluminescent Assay. J Biolumin Chemilum. 1995; 10:63-67.

[13] Miedzobrodzki J, Kaszycki P. Effect of Staphylococcus aureus serine proteinase on the respiratory burst in phagocytic cells in vitro. Acta Microbiol Pol. 2000;49:237-242.

[14] McEver RP. Adhesive interactions of leukocytes, platelets, and the vessel wall during hemostasis and inflammation. Thromb Haemost. 2002;86:746-756.
[15] Gear ARL, Camerini D. Platelet Chemokines and Chemokine Receptors: Linking Hemostasis, Inflammation, and Host Defense. Microcirculation. 2003;10:335-350.

[16] Peters MJ, Dixon G, Kotowicz KT, Hatch DJ, Heyderman RS, Klein NJ. Circulating platelet-neutrophil complexes represent a subpopulation of activated neutrophils primed for adhesion, phagocytosis and intracellular killing. Br J Haematol. 1999;106:391-399.

[17] Tsuji T, Nagata K, Koike J, Todoroki N, Irimura T. Induction of superoxide anion production from monocytes and neutrophils by activated platelets through the P-selectin-sialyl Lewis X interaction. J Leukocyt Biol. 1994;56:583-587.

[18] Stanislawska J, Interewicz B, Olszewski WL. Immune response of host leukocytes for bacterial antigens. Post Mikrobiol. 2003;42:301-317.

[19] Moon DG, van der Zee H, Weston IK, Gudewicz PW, Fenton JW, Kaplan JE. Platelet modulation of neutrophil superoxide anion production. Thromb Haemost. 1990;63:91-96.

[20] Jugeau S, Tenaud I, Knol AC, Jarrousse V, Quereux G, Khammari A, Dreno B. Induction of toll-like receptors by Propionibacterium acnes. Br J Dermatol. 2005;153:1105-1113.

[21] Plonka PM, Chlopicki S, Wisniewska M, Plonka BK. Kinetics of increased generation of $\mathrm{NO}$ in endotoxaemic rats as measured by EPR. Acta Biochim Pol. 2003;50:807-813.

[22] Iuliano L, Pedersen JZ, Pratico D, Rotilio G, Violi F. Role of hydroxyl radicals in the activation of human platelets. Eur $J$ Biochem. 1994;221:695-704.

[23] Dore M. Platelet-leukocyte interactions. Amer Heart J. 1998; 135:S146-S151.

[24] Chlopicki S, Lomnicka M, Gryglewski RJ. Obligatory role of lipid mediators in platelet-neutrophil adhesion. Thromb Res. 2003;15:287-292. 